\title{
La prise en compte du risque crue en région parisienne par EDF-GDF
}

\author{
The taking into account by EDF-GDF of the risk of flood \\ in the Paris area
}

\author{
M. Lion \\ E.D.F.-D.E.P.T. Ile-de-France Ouest \\ J.-L. Losfeld \\ E.D.F.-D.E.P.T. Ile-de-France Ouest \\ X. Beraud-Dupalis \\ E.D.F.-G.D.F. Services à Paris
}

The structure of the electricity supply in the Ile-de-France region and particularly in Paris is recalled. The region is so densely populated that a presentation of the effects of a flood like the one in 1910 on Paris electricity network and on our customers will be carried out. This presentation will show the consequences of such a flood, as also the dispositions taken as a prevention, during the flood, and after it.

In Ile-de-France and in Paris, the main consequence of a flood like in 1910 will be the loss of power for 550,000 customers, i.e. approximately 900,000 inhabitants.

The objectives of Electricite de France in case of a flood like that will be :

- to confine at the maximum the losses of power to the flooded areas,

- to set up as many means of supplying power as possible (generating sets, provisional electricity network...),

- to manage in real time the information on flood levels,

- to keep in touch with the crisis management team set up by the authorities,

- to inform our customers on the evolution of their situation by all the possible means.

\section{ALIMENTATION ÉLECTRIQUE DE PARIS}

- 1.1 Transport de l'énergie électrique vers l'Ile-deFrance et vers Paris

L'alimentation en énergie électrique de l'Ile-de-France s'appuie sur huit postes de transformation $400 \mathrm{kV} / 225 \mathrm{kV}$ répartis sur une double boucle de réseaux $400 \mathrm{kV}$ et $225 \mathrm{kV}$ située dans les départements de la deuxième couronne. (cf. fig. l)
L'énergie est transportée jusqu'à des postes de livraison 225 $\mathrm{kV} / 20 \mathrm{kV}$ par des lignes $225 \mathrm{kV}$ pour desservir l'ensemble de la banlieue parisienne. Elle est transportée d'autre part jusqu'à 13 postes de répartition $225 \mathrm{kV}$ situés en proche banlieue. Ces lignes $225 \mathrm{kV}$ sont souvent regroupées en couloir.

L'alimentation de la ville de Paris est réalisée, à partir de ces 13 postes de répartition $225 \mathrm{kV}$, par des radiales $225 \mathrm{kV}$ pénétrant vers le cœur de la ville, constituées de liaisons souterraines $225 \mathrm{kV}$ qui injectent la puissance sur le réseau de distribution $20 \mathrm{kV}$ par l'intermédiaire de 34 postes de transformation $225 \mathrm{kV} / 20 \mathrm{kV}$. 


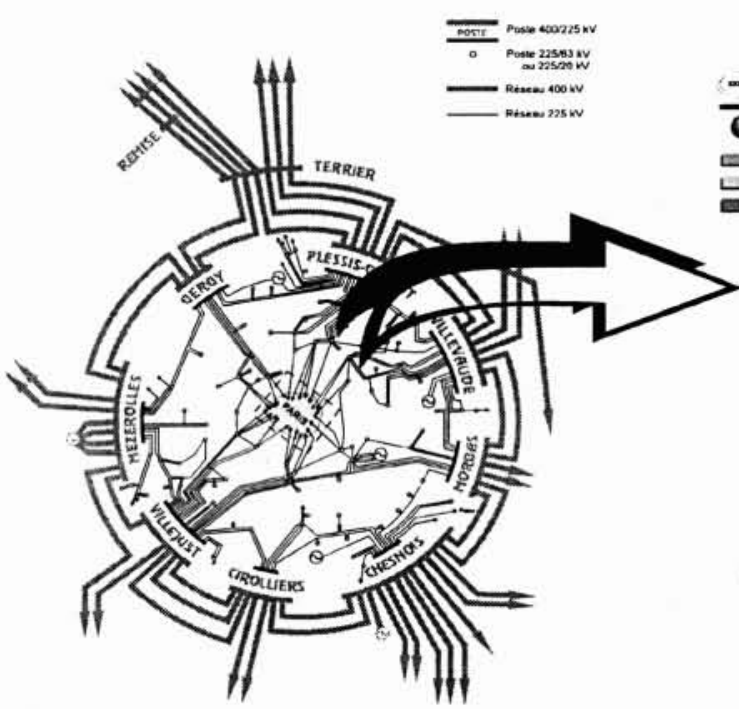

1. Alimentation $400 \mathrm{kV}$ et $225 \mathrm{kV}$ de l'Ile-de-France.

\subsection{Distribution de l'énergie électrique à Paris}

Le réseau de distribution $20 \mathrm{kV}$, qui irrigue Paris, est constitué de trois couronnes concentriques indépendantes (cf. fig. 2):

- la couronne extérieure située à la périphérie,

- la couronne médiane en cours de réalisation,

- la couronne intérieure proche du centre.

Les postes $225 \mathrm{kV} / 20 \mathrm{kV}$ d'une même couronne sont reliés par quatre ensembles de 6 câbles $20 \mathrm{kV}$ (appelés artères) qui assurent la desserte en énergie électrique des différents quartiers à travers environ 6000 postes de transformation $20 \mathrm{kV} / 400 \mathrm{~V}$ et un réseau $400 \mathrm{~V}$ associé.

Chacune des artères est alimentée indifféremment par l'un ou l'autre des postes-sources auquel elle est raccordée, le choix s'effectuant en fonction des disponibilités d'ouvrages et des charges du moment.

\subsection{Particularités de l'alimentation en énergie élec- trique de Paris}

De manière globale Electricité de France alimente :

- en Ile-de-France 5,8 millions de clients qui représentent 10,8 millions d'habitants. La densité de population d'Ile-deFrance est de 900 habitants $/ \mathrm{km}^{2}$,

- dont à Paris 1,5 millions de clients qui représentent 2,2 millions d'habitants. La densité de population de Paris est de 20500 habitants $/ \mathrm{km}^{2}$.

Nous pouvons remarquer l'extrême densité de la population à Paris, ce qui nous amènera à nous intéresser plus particulièrement aux conséquences d'une crue de type 1910 à Paris.

Du fait de cette densité (place pour les ouvrages électriques limitée), la structure d'alimentation de Paris intramuros est spécifique : un défaut sur une liaison radiale $225 \mathrm{kV}$ se traduit par la coupure de tous les postes raccordés en cascade sur celle-ci et par conséquent par la mise hors tension d'un grand nombre de transformateurs. La structure de Paris prévoit donc de conserver une marge de sécurité suffisante sur la puissance de transformation des postes $225 \mathrm{kV} / 20 \mathrm{kV}$ et sur la capacité des artères $20 \mathrm{kV}$, pour réalimenter toute la clientèle lors de la défaillance ou l'indisponibilité d'un ou plusieurs ouvrages $225 \mathrm{kV}$.
Ainsi la structure électrique de Paris est dimensionnée afin de faire face à l'indisponibilité simple ou double de deux liaisons radiales voisines.

\section{II —EAU ET ÉLECTRICITÉ (RÉACTION DU RÉSEAU À UNE CRUE)}

\subsection{Effet de l'eau sur le réseau électrique}

L'eau pure et l'électricité font très bon ménage ! Malheureusement, une eau est rarement pure et contient presque toujours des impuretés en particulier lors de crues. Dans ce cas l'eau devient conductrice et provoque un court-circuit entre les phases du réseau électrique. Les effets sont les mêmes à tout niveau de tension du réseau.

Chez le particulier, un court-circuit dû à l'eau provoque immédiatement l'ouverture du disjoncteur du client.

Sur le réseau Basse Tension $(400 \mathrm{~V})$, un court-circuit dû à l'eau provoque immédiatement la fusion du fusible en tête du départ dans le poste de Distribution Publique.

Sur le réseau Moyenne Tension $(20 \mathrm{kV})$, un court-circuit dû à l'eau provoque immédiatement l'ouverture du disjoncteur du câble d'artère ou de l'artère et en dernière extrémité du demi-poste-source.

Sur le réseau Haute Tension $(225 \mathrm{kV})$, un court-circuit dû à l'eau provoque immédiatement l'ouverture du disjoncteur $225 \mathrm{kV}$ de la radiale situé dans le poste de répartition $225 \mathrm{kV}$ situé en proche banlieue.

En conséquence, il est nécessaire pour Electricité de France de circonscrire au plus près les défauts dus à une crue afin d'éviter une généralisation de l'incident.

\subsection{Impact d'une inondation sur le réseau de Trans- port 225 et $63 \mathrm{kV}$ d'Ile de France}

Des dispositions sont prises visant à protéger les ouvrages contre les agressions climatologiques.

Tous les ouvrages constitutifs du réseau électrique sont étudiés et réalisés pour être insensibles à un ensemble d'hypothèses générales représentatives des contraintes auxquelles ils seront soumis au cours de leur exploitation. 
Le risque d'inondation fait partie des risques examinés, spécialement pour les ouvrages souterrains et des dispositions sont prises en particulier pour les rendre étanches aux infiltrations et aux ruissellements consécutifs aux orages.

Dans ce recensement des intempéries et autres agressions auxquelles les ouvrages peuvent être soumis, il y a lieu, pour choisir le niveau de protection à envisager, de prendre en considération la probabilité d'apparition du risque, l'ampleur des répercussions sur l'alimentation des clients ainsi que le coût des protections envisageables.

Sauf, en effet, à augmenter le coût de l'électricité très audelà des montants admissibles par la collectivité, les ouvrages du réseau ne peuvent pas être dimensionnés pour rester totalement indemnes face à tous événements y compris les moins probables. Une crue exceptionnellement sévère sort ainsi manifestement du cadre des phénomènes dont les ouvrages du réseau électrique pourraient sortir totalement indemnes.

En Ile de France, on peut noter que les lignes aériennes, compte tenu de la distance par rapport au sol de leurs parties sous tension, et les lignes souterraines, de leur étanchéité, sont pratiquement insensibles à la montée des eaux et peuvent être maintenues sous tension dans le scénario de crue de type 1910.

Par contre les postes $225 \mathrm{kV} / 20 \mathrm{kV}$ et les postes de Distribution Publique $20 \mathrm{kV} / 400 \mathrm{~V}$, de par leurs parties nues sous tension, sont très sensibles à la montée des eaux.

Dès lors que le terrain du poste $225 \mathrm{kV} / 20 \mathrm{kV}$ est inondé, il y a lieu de mettre hors tension les circuits électriques auxiliaires. Dans ces conditions, il n'est alors plus possible de réaliser les manœuvres d'exploitation et les transformateurs
$225 \mathrm{kV} / 20 \mathrm{kV}$, privés de leur refroidissement, ne peuvent plus être exploités. En revanche, les installations de transit de puissance peuvent, dans la plupart des cas, être maintenues sous tension et il est possible de constituer des « files de lignes " permettant le transit d'énergie au travers des postes inondés et de maintenir dans ces conditions dégradées, le fonctionnement du réseau.

\subsection{Impact d'une inondation sur les ouvrages élec- triques de la ville de Paris}

Les principales caractéristiques des ouvrages électriques de Paris sont les suivantes :

- l'ensemble des réseaux $(225 \mathrm{kV}, 20 \mathrm{kV}, 400 \mathrm{~V})$ est en souterrain,

- $55 \%$ des postes de Distribution Publique sont enterrés sous chaussée, le reste étant en immeuble (rez-de-chaussée ou sous-sol),

— les Postes Sources sont soit totalement sous chaussée soit partiellement.

Ces ouvrages sont vulnérables aux phénomènes de crues.

Un important programme de travaux a été entrepris pour restructurer le réseau afin de limiter les conséquences d'un incident de grande ampleur:

- radialisation des réseaux Basse Tension pour une souplesse de conduite en cas d'incident,

- tronçonnement des artères $20 \mathrm{kV}$ par des interrupteurs télécommandés permettant d'isoler au plus juste les zones en défaut,

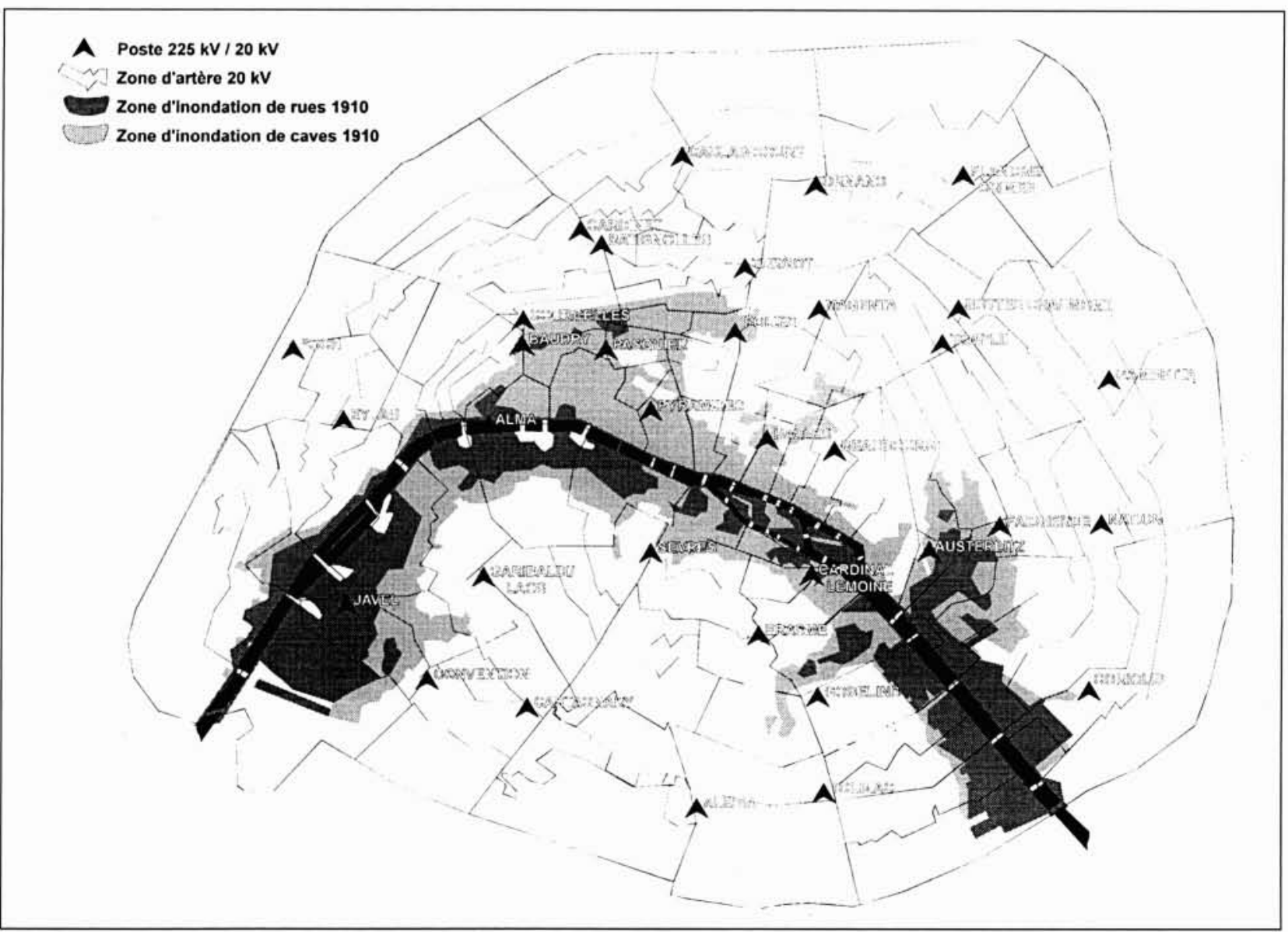

3. Superposition des zones d'inondation de rues et de caves en 1910 à Paris avec l'emplacement des postes $225 \mathrm{kV} /$ $20 \mathrm{kV}$ et les zones d'actions d'artères $20 \mathrm{kV}$. 
- politique d'implantation de postes de Distribution Publique $(20 \mathrm{kV} / 400 \mathrm{~V})$ en rez-de-chaussée d'immeuble (pour éviter les problèmes de ruissellement en cas d'orage),

- interrupteurs $20 \mathrm{kV}$ de sécurisation en sortie de galerie de câbles,

- les postes-sources souterrains sont conçus de manière étanche.

Ces travaux s'achèveront à l'horizon 2000.

Ces travaux ne permettront malheureusement pas de maintenir l'alimentation dans les zones où les rues et les caves sont inondées mais de circonscrire au plus juste les parties du réseau en défaut. L'étanchéité totale de notre réseau pour ce type de crue ne pourrait être réalisée qu'à des coûts exorbitants ayant des conséquences importantes sur les factures de nos clients.

\section{III —CONSÉQUENCES D'UNE CRUE DE TYPE 1910}

\subsection{Zones d'inondation}

Comme il a été vu plus haut, il résulte de la crue non pas l'indisponibilité du réseau de Transport mais sa fragilisation par la suppression des possibilités de manœuvres dans les postes Haute Tension inondés et la réalisation de schémas en files simplifiés.

Concernant ces postes en Ile de France, 37 postes situés dans la zone de crue subiraient une inondation et les dégâts sur les installations nécessiteraient une remise en état plus ou moins importante à la décrue.

Pour ce qui concerne Paris Intra-Muros, la figure 3 représente les zones d'inondation des rues et des caves probables en cas d'atteinte des niveaux de crue de 1910. Une comparaison avec l'emplacement des postes-sources $(225 \mathrm{kV} /$ $20 \mathrm{kV}$ ) et des zones d'action des artères $20 \mathrm{kV}$ nous permet de prévoir les zones susceptibles d'être privées d'alimentation.

\subsection{Zones susceptibles d'être privées d'électricité à Paris}

L'analyse des différents sites touchés nous permet de tirer les conclusions suivantes (cf. fig. 4).

POSTES $225 \mathrm{kV} / 20 \mathrm{kV}$

- 6 postes $225 \mathrm{kV} / 20 \mathrm{kV}$ sont hors service avec transit d'énergie $20 \mathrm{kV}$ impossible (Baudry, Pasquier, Pyramides, Halles, Austerlitz et Javel),

- 1 poste $225 \mathrm{kV} / 20 \mathrm{kV}$ est hors service avec transit d'énergie $20 \mathrm{kV}$ possible (Folies),

- 6 postes $225 \mathrm{kV} / 20 \mathrm{kV}$ sont maintenus en service sous réserve de défenses locales pour contrôler les pénétrations d'eau par les galeries (Courcelles, Cardinal-Lemoine, Convention, Faidherbe, Garibaldi et Alma),

- les 21 autres postes sources ne sont pas touchés.

ARTĖRES $20 \mathrm{kV}$ (après fonctionnement des appareils de tronçonnement du réseau $20 \mathrm{kV}$ )

- 35 artères $20 \mathrm{kV}$ sont mises hors tension totalement,

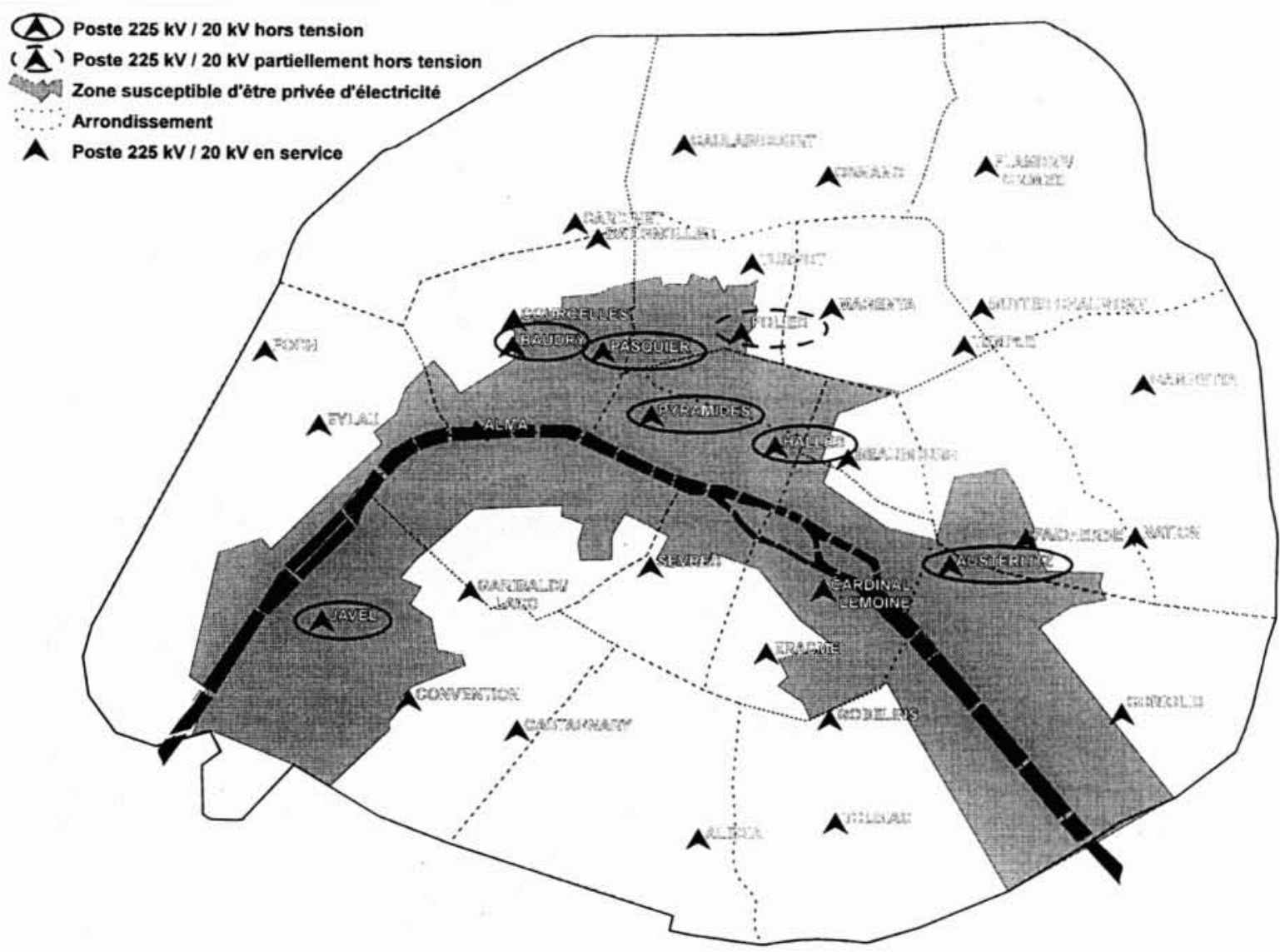

4. Postes $225 \mathrm{kV} / 20 \mathrm{kV}$ mis hors tension et zone susceptible d'être privée délectricité lors d'une crue de type 1910 à Paris. 
- 25 artères $20 \mathrm{kV}$ sont mises hors tension partiellement par fonctionnement d'interrupteurs de tronçonnement, - les 74 autres artères $20 \mathrm{kV}$ ne sont pas touchées.

En conclusion, en cas de crue de type 1910, le nombre de clients privés d'électricité à Paris est estimé à 355000 , ce qui représente environ 500000 habitants. Cela signifie qu'environ 1/4 de la population parisienne est touché.

\section{GESTION DE LA CRISE}

\subsection{Exploitation du préavis de crue}

Au moment de l'apparition des signes précurseurs d'une crue de grande ampleur de type 1910, le préavis doit être mis à profit pour rassembler et organiser les moyens humains et matériels et préparer le réseau dans des conditions permettant de faire face à la crise.

Des plans de crise préétablis, et régulièrement testés en liaison avec les autorités, prévoient l'organisation «de crise " à mettre en place et décrivent les liaisons entre les PC de crise d'EDF et les autorités administratives. Ils recensent également les moyens de secours disponibles.

Plusieurs tâches sont à mener pendant la période de préavis : - la mise sous surveillance des installations menacées en vue de limiter le plus possible l'étendue et la gravité des dégâts, de procéder dans les meilleures conditions, le moment venu, à la mise hors tension des « auxiliaires » et de la partie inexploitable des installations, et de réaliser les conditions de la «sécurité électrique »,

- la préparation de la mise en place des schémas de réseau optimaux préétablis permettant de faire face le mieux possible à la fragilisation du réseau,

- le rassemblement et la préparation des moyens recensés dont EDF dispose sur le territoire (et notamment les groupes électrogènes mobiles) pour contribuer au mieux à l'organisation des secours concernant les clients privés d'électricité.

\subsection{Gestion de la crise pendant la crue dans la ville de Paris}

Les clients situés hors zone de crue sont presque normalement alimentés. En raison de la structure du réseau parisien, chaque client est alimenté par des câbles raccordés systématiquement à deux postes $225 \mathrm{kV} / 20 \mathrm{kV}$ différents. Dans la plupart des cas, en raison de la distance entre ces deux postes, si l'un des deux doit, du fait de la crue, être mis hors tension et rendu indisponible, le deuxième sera à l'abri de la crue et pourra assurer l'alimentation du client, pour autant que ses propres installations seront elles-mêmes préservées de l'inondation.

Une cellule de crise sera mise en place pour assurer la coordination des moyens mis en œuvre et l'information des
Autorités. Un suivi des informations sur les hauteurs de crue sera mis en place afin de tenir à jour une carte des ouvrages en corrélation avec les zones de crues.

L'installation électrique des clients inondés doit être séparée du réseau.

Dans l'état des techniques, les installations électriques d'entrée d'immeuble ne sont pas de façon générale étanches à l'inondation des caves et, pour des raisons techniques comme de sécurité, elles doivent être séparées du réseau d'alimentation (et le sont en tout état de cause automatiquement en raison de l'action des systèmes de sécurité).

La possibilité de préserver l'alimentation électrique des étages supérieurs non inondés relève alors de l'emploi de groupes électrogènes autonomes dont la mise en œuvre nécessite un examen technique au cas par cas.

Comme indiqué plus haut, EDF aura rassemblé la totalité des groupes électrogènes dont elle dispose sur le territoire, et, en relation avec les autorités, les mettra à la disposition de la collectivité pour assurer les alimentations de secours.

Ces interventions d'EDF seront coordonnées en liaison avec les pouvoirs publics par le moyen des organisations de crise coordonnées dans des conditions préalablement testées et contrôlées.

Un souci majeur d'Electricité de France sera d'informer en temps réel l'ensemble de sa clientèle privée d'électricité sur l'état d'avancement des possibilités de réalimentation. Ce même souci d'information se retrouvera vis-à-vis des autorités concédantes.

\subsection{Gestion de la crise après la crue}

A l'issue de la crise, il y a lieu de procéder à la réparation des dommages subis par les ouvrages.

Les ouvrages peu touchés (ouvrages aériens dont le niveau d'inondation n'aura pas dépassé $50 \mathrm{~cm}$ ) seront nettoyés en quelques jours, alors qu'il y aura lieu de procéder à la remise en état ou au remplacement des matériels largement submergés dans les postes souterrains.

Selon les cas, le budget à prévoir sera de quelques centaines de $\mathrm{kF}$ à quelques MF par ouvrage touché.

Electricité de France mettra tout en œuvre pour nettoyer les installations, remettre en état les appareils réparables, approvisionner et mettre en place les matériels de remplacement des appareils avariés, pour rétablir au plus vite la totalité du Service Public.

Les travaux de remise en ordre de la totalité des installations pourront s'étaler jusqu'à un an en fonction de l'importance des événements.

Chaque événement, même localisé et mineur, donne lieu à un retour d'expérience qui est capitalisé pour se prémunir face à des phénomènes de plus grande ampleur. De plus chacun d'eux permet d'augmenter l'efficacité et la réactivité de nos équipes. 